\title{
Predictors of Implantable Cardioverter-Defibrillator Use in Patients with Ischemic Cardiomyopathy
}

\author{
Ryan Kelly ${ }^{1}$, Karen J Buth ${ }^{1}$, Olivier Heimrath ${ }^{1}$, Magdy Basta ${ }^{2}$ and Jean-Francois Legare ${ }^{1, *}$
}

Departments of Surgery ${ }^{1}$ and Medicine ${ }^{2}$, Dalhousie University, Halifax, Nova Scotia

\begin{abstract}
Objectives: The objective of this study was to identify and examine ICD utilization in a large group of eligible coronary artery bypass grafting $(\mathrm{CABG})$ patients with impaired left ventricular function.

Methods: We conducted a retrospective study of ICD eligible patients who had previously undergone CABG surgery between March 1, 1995 and June 30, 2008 at a single tertiary care institution. All patients with a pre-operative left ventricular ejection fraction $(\mathrm{LVEF}) \leq 35 \%$ were considered ICD eligible. The events of interest were ICD implantation and mortality, based on administrative data linkage.

Results: A total of 1,169 out of 11,931 CABG patients operated on during the same period had LVEF $\leq 35 \%$ and were defined as ICD eligible (mean $\mathrm{EF}=27.3 \%+/-6.4 \%$ ). Of these eligible patients, only 101 received an ICD during followup $(8.6 \%)$. The median time to implant was 255 days (14-1078). The single variable that independently predicted eventual ICD implantation was a history of arrhythmia (OR = 7.4; CI, 4.4-12.2). The variables that predicted not having an ICD implanted during follow-up included the need for urgent CABG $(\mathrm{OR}=0.5$; CI, 0.2-0.9), age $>70$ years $(\mathrm{OR}=0.5$; CI, 0.3-0.8), female gender $(\mathrm{OR}=0.2$; $\mathrm{CI}, 0.1-0.6)$, or having chronic obstructive lung disease $(\mathrm{OR}=0.5$; $\mathrm{CI}, 0.3-0.8)$. As a data validation step, a series of consecutive patient records were reviewed $(n=80)$ showing that fewer than $23 \%$ underwent appropriate follow-up EF assessment post revascularization.

Conclusion: Our findings suggest that CABG patients with ischemic cardiomyopathy have low rates of ICD utilization. This is particularly evident among females and elderly patients. Furthermore our data suggests that few patients postrevascularization undergo follow-up EF assessment despite current guidelines likely contributing to the low rates of ICD utilization.
\end{abstract}

Keywords: Implantable cardioverter-defibrillator, left ventricular ejection fraction, coronary artery bypass graft, arrhythmia

\section{BACKGROUND}

Implantable cardioverter-defibrillator (ICD) devices have played a key role in anti-arrhythmic intervention. These devices are currently used for the prevention of fatal arrhythmias, particularly in patients with ischemic disease [1]. Historically, ICD devices were used solely for the secondary prevention of recurrent ventricular fibrillation (VF) and ventricular tachycardia (VT) [1-4]. However, the indication for ICD implantation has evolved over the past decade to include the prevention of potentially lethal arrhythmias in patients with ventricular dysfunction who are otherwise asymptomatic [5-8]. This shift towards primary prevention was due in large part to the findings of several large randomized control trials, which have reported significant mortality risk reduction with the prophylactic use of ICD therapy $[5,6,8$ 10].

Current Canadian Cardiovascular Society (CCS) recommendations state that prophylactic ICD therapy should be considered for patients with ischemic heart disease with

*Address correspondence to this author at the New Halifax Infirmary, Division of Cardiac Surgery, 1796 Summer St. Rm 2269, Halifax, Nova Scotia, Canada, B3H 3A7; Tel: (902) 473-3808; Fax: (902) 473-4448;

E-mail: kellyrp@dal.ca or without mild to moderate heart failure symptoms and a reduced left ventricular ejection fraction (LVEF). Ejection fraction should be measured at least 1 month after myocardial infarction (MI) and at least 3 months following coronary revascularization (class I, level A). ICD therapy may also be considered in patients with non-ischemic cardiomyopathy present for at least 9 months, New York Heart Association (NYHA) functional class II to III heart failure, and an LVEF $\leq 30 \%$ (class IIa, level B) or an LVEF of $31 \%$ to $35 \%$ (class IIb, level C). Additionally, an ICD may be considered in patients with ischemic heart disease, previous MI, LV dysfunction (LVEF of $31 \%$ to $35 \%$ ) measured at least 1 month after MI and 3 months after coronary revascularization and with inducible VF / sustained VT at electrophysiology study (class IIa, level B), or without an electrophysiology study (class IIb, level C evidence) [11-14]. It is important to note that ICD devices should not be implanted in patients who exhibit NYHA class IV heart failure if they are not candidates for transplantation, or those patients that are not expected to survive with adequate functional status for more than a year [12-15].

Despite these recommendations, however, ICD devices appear underutilized among candidates eligible for primary prevention of lethal arrhythmias [16-19]. Thus, for the pur- 
pose of this study we combined the above recommendations to evaluate the utilization of ICD therapy at our institution in patients with ischemic cardiomyopathy who have undergone coronary artery bypass grafting (CABG) surgery, with mildmoderate heart failure symptoms, with left ventricular dysfunction $(\mathrm{LVEF} \leq 35 \%)$. Patients who met these criteria were deemed to be eligible for primary prevention with an ICD. After determining ICD utilization at our center we attempted to identify discrete patient characteristics that were independent predictors of receiving an ICD post surgical revascularization. Lastly, we also examined the long-term survival of eligible patients who received an ICD compared to eligible patients who did not receive a device.

\section{METHODS}

\section{Patients}

We examined all consecutive adult patients who underwent surgical coronary revascularization (CABG) at the Queen Elizabeth II Health Sciences Center (QEII HSC) between March 1, 1995 and June 30, 2008. From this group, only patients with LVEF $\leq 35 \%$ were included. These patients were then divided into two groups: a 'CABG + ICD' group who received an ICD following $\mathrm{CABG}$, and a ' $\mathrm{CABG}$ only' group who did not receive an ICD at any point in time during follow-up after CABG. Determination of LVEF was based on preoperative echocardiography, left ventricular angiography, and nuclear imaging when available.

Patients eligible for CABG surgery at our institution have traditionally been stratified based on a weekly peer review process involving cardiologists, cardiac surgeons, and cardiac radiologists. Individual patients were queued for surgery based on objective criteria as previously described [20]. All patients were assigned the first available surgeon. Isolated CABG surgery was performed in $79 \%$ of patients. The remainder of patients underwent a combination of CABG and other procedures, which include maze, aortic and mitral valve replacement or repair.

Patients were evaluated individually for the need for ICD therapy without a predetermined referral pattern. As such, patients were determined to be eligible on an individual basis following the recommendation from a group of collaborating electrophysiology specialists (EPS) practicing at the QEII HSC. Standard CCS criteria for ICD implantation were followed $[11,14]$. The types of ICD devices used at our institution were reflective of hospital contracts with the following manufacturers: Guidant, St. Jude, and Medtronic and included single, dual, and bi-ventricular lead devices. All devices were implanted in an operating room by a cardiovascular surgeon and an EPS physician

\section{Data Sources}

The QEII HSC is the only tertiary care center in Nova Scotia, servicing over 1 million people, and is the site for all cardiac catheterization procedures and surgical interventions in the province. In a given year, over 1,200 adult cardiac surgeries are preformed at the QEII HSC. The Maritime Heart Center Cardiac Surgery Registry Database captures pre-operative, intra-operative, and in-hospital data from all $\mathrm{CABG}$ operations preformed in this province.
Data linkage between Maritime Heart Center Database and Population Health Research Unit (PHRU) at Dalhousie University was carried out using unique patient identifiers. Through PHRU we were able to access Nova Scotia Vital Statistics (mortality data) and the Canadian Institute for Health Information (CIHI) Discharge Abstract Database (DAD) to obtain all-cause mortality data and long-term follow-up data for the patients in the present study. All data was reported as aggregate, which restricted access to individual patient information.

\section{Variable Selection}

Patients were grouped based on age as follows: $<60,60$ $-69,70-79,>80$. Other pre-operative variables included, gender, smoking history, recent MI (MI < 7 days before CABG), hypertension, congestive heart failure, diabetes, peripheral vascular and cerebrovascular disease, chronic obstructive pulmonary disease, stenosis of the left main coronary artery $>50 \%$, renal failure (pre-operative serum creatinine of $>176 \mu \mathrm{mol} / \mathrm{L})$, ventricular arrhythmia, severity of coronary artery disease (single versus double or left main disease versus triple), complexity of procedure (CABG alone versus $\mathrm{CABG}$ with additional procedures including valve or maze), incidence of redo surgery, and urgency of surgery. Urgency of surgery was determined and recorded at the time of surgery and was stratified as follows: Elective (stable, waiting at home), In-House Urgent (requiring hospitalization until the time of surgery), Urgent (requiring surgery within 24 hours for symptoms or anatomy), and Emergent/Emergent Salvage (no delay, first available operating room).

\section{Statistical Analysis}

Univariate analyses of pre-operative, intra-operative, and post-operative in-hospital variables was carried out using Chi-square tests for dichotomous variables, two tailed t-tests for normally distributed continuous variables, and Wilcoxon rank sum tests for continuous variables that were not normally distributed. Variables that were shown to exhibit difference between the 'CABG + ICD' and 'CABG only' groups as well as clinically relevant characteristics were examined as variables in multivariate logistic regression analysis. Within the multivariate model an estimate was generated from each variable from which an odds ratio, p-value, and 95\% CI was calculated. A receiver operating characteristic curve was calculated as a measure of sensitivity and specificity for our logistic regression model. Kaplan Meier survial curves for our 'CABG + ICD' and 'CABG only' groups were produced. A Cox proportional hazards ratio model was also produced to determine if ICD therapy was an independent predictor of improved survial. We included age, gender, preop renal failure, arrhythmia, redo surgery, urgent or emergent status, chronic obstructive pulmonary disease (COPD), and cerebrovascular accident in our model in order to generate an adjusted hazard ratio. All statistical analysis was performed using SAS software version 9.1 (SAS, Cary, North Carolina).

\section{Data Validation Group}

In a limited group of consecutive patients (2006-2007) we preformed a thorough chart review. The primary objective of this abstraction was to determine the rate of appropri- 
ate LVEF assessment post-revascularization. Second to this, we wanted to determine the number of patients who showed improvement in $\mathrm{EF}>35 \%$ which, according to current guidelines, would negate their eligibility for primary prevention with ICD therapy.

\section{RESULTS}

Between March 1, 1995 and June 30, 2008 a total of 1,169 out of $11,931(9.8 \%)$ patients undergoing CABG surgery in Halifax were found to have a pre-operative left ventricular ejection fraction $(\mathrm{LVEF})$ of $\leq 35 \%$ (mean $\mathrm{LVEF}=$
$27.3 \%+/-6.4 \%)$. These ischemic cardiomyopathy patients were defined as the ICD eligible group for the purpose of the present study. Of ICD eligible patients, 101 received an ICD some time after their CABG surgery (8.6\%). Eight patients received an ICD prior to $\mathrm{CABG}$ and were excluded from the analysis. Patients were divided in 2 groups based on their eventual implantation of an ICD. Those that received an ICD ('CABG + ICD' group) were compared to those that did not ('CABG only' group). Patient demographics and comorbidities of the two groups are shown in Table 1. Patients who ultimately received ICD therapy were younger, more likely to be male, have a history of ventricular arrhythmias,

Table 1. Pre- and Intra-Operative Characteristics among CABG Patients with LVEF $\leq 35 \%$

\begin{tabular}{|c|c|c|c|}
\hline Characteristic & $\begin{array}{c}\text { CABG + ICD } \\
n=101(\%)\end{array}$ & $\begin{array}{c}\text { CABG only } \\
\mathrm{n}=1068(\%)\end{array}$ & p -Value \\
\hline Age $<60$ & $39(39)$ & $262(24)$ & \multirow{4}{*}{$<0.01$} \\
\hline Age $60-69$ & $36(36)$ & $328(31)$ & \\
\hline Age $70-79$ & $23(23)$ & $369(35)$ & \\
\hline Age $\geq 80$ & $3(3)$ & $109(10)$ & \\
\hline Female Gender & $6(6)$ & $239(22)$ & $<0.01$ \\
\hline Smoking & $85(85)$ & $817(77)$ & 0.08 \\
\hline $\mathrm{MI}<7 \mathrm{~d}$ & $13(13)$ & $172(16)$ & 0.47 \\
\hline Hypertension & $61(60)$ & $657(62)$ & 0.83 \\
\hline $\mathrm{CHF}$ & $54(54)$ & $624(58)$ & 0.34 \\
\hline Diabetes & $42(42)$ & $471(44)$ & 0.68 \\
\hline PVD & $25(25)$ & $274(26)$ & 0.91 \\
\hline CVD & $16(16)$ & $171(16)$ & 1.00 \\
\hline COPD & $14(14)$ & $268(25)$ & 0.01 \\
\hline LM Stenosis $>50 \%$ & $24(24)$ & $278(26)$ & 0.72 \\
\hline Renal Failure & $7(7)$ & $164(15)$ & 0.02 \\
\hline Ventricular Arrhythmia & $37(37)$ & $83(8)$ & $<0.01$ \\
\hline Single Vessel Disease & $1(1)$ & $54(5)$ & \multirow{3}{*}{0.18} \\
\hline Double Vessel Disease & $18(18)$ & $180(17)$ & \\
\hline Triple Vessel Disease & $82(82)$ & $834(78)$ & \\
\hline CABG Surgery Only & $80(79)$ & $848(79)$ & \multirow{2}{*}{1.00} \\
\hline $\mathrm{CABG}+$ Other Procedure & $21(21)$ & $220(21)$ & \\
\hline Redo & $12(12)$ & $90(8)$ & 0.27 \\
\hline Elective Status & $34(34)$ & $297(28)$ & \multirow{4}{*}{0.35} \\
\hline IHU Status & $43(42)$ & $431(40)$ & \\
\hline Urgent Status & $16(16)$ & $240(23)$ & \\
\hline Emergent Status & $8(8)$ & $100(9)$ & \\
\hline
\end{tabular}

Smoking - includes past or present, MI - myocardial infarction, CHF - congestive heart failure, PVD -peripheral vascular disease, CVD - cerebrovascular disease, COPD - chronic obstructive pulmonary disease, Redo - reoperation for cardiac surgery, IHU - in hospital urgent, LVEF - Left ventricular ejection fraction. 
and were less likely have COPD or be in renal failure. In fact, only 6 of 245 eligible females with ischemic cardiomyopathy received an ICD during follow-up (2.4\%).

Freedom from ICD implantation in eligible patients was $95 \%$ at year $1,93 \%$ at year 3 , and $91 \%$ at year 5, suggesting low rates of utilization (Fig. 1). The median time to implant was 255 days post revascularization (IQR 14 - 1078 days) with $39 \%$ of patients receiving an ICD within 90 days of CABG surgery. This is in contrast to the increasing number of ICD implants performed in Halifax over the past decade (Fig. 2). Similarly, the proportion of ICD implants in patients with a history of surgical revascularization has also steadily increased over the same time period from $0 \%$ in 1997 to $10.5 \%$ of all ICD implants in 2007.

A multivariate logistic regression analysis was used to correct differences between the 'CABG + ICD' and 'CABG only' groups and to identify predictors of ICD implantation in ischemic cardiomyopathy patients. A history of preoperative arrhythmia was the only variable found to independently predict eventual ICD implantation $(\mathrm{OR}=7.4 ; 95 \%$ confidence interval, 4.4-12.2) (Table 2). Independent variables that predicted patients not having an ICD implanted post $\mathrm{CABG}$ included having an urgent or emergent surgery on their original presentation $(\mathrm{OR}=0.5 ; \mathrm{CI}, 0.2-0.9)$, age > 70 years $(\mathrm{OR}=0.5 ; \mathrm{CI}, 0.3-0.8)$, being female $(\mathrm{OR}=0.2$; CI, 0.1-0.6), or having COPD (OR = 0.5; CI, 0.3-0.8) (Table 2). The receiver operating characteristic for the model was $78 \%$.

One should note that long-term follow up data for mortality analysis was available for 61 of our 101 patients. The 40 patients lost to analysis failed to link to the Nova Scotia Vital Statistics Database as the majority of these were Maritime patients from outside of Nova Scotia. The survival benefit for patients with an ICD is illustrated in Fig. (3). Survival at 1,3 and 5 years was $95 \%, 89 \%$, and $83 \%$ for the 'CABG+ICD' group compared to $92 \%, 83 \%$ and $70 \%$ for the 'CABG only' group ( $\log$ rank $\mathrm{p}=0.02)$. At 5 years, patients who received an ICD had a relative risk reduction of death of $45 \%$. Using Cox proportional hazards modeling, the unadjusted hazard ratio for ICD implantation was $0.49(95 \%$ CI, $0.28-0.74 ; \mathrm{p}=0.02)$, and with adjustment, the hazard ratio decreased to $0.63(95 \% \mathrm{CI}, 0.33-1.20 ; \mathrm{p}=0.16)$. Fig. (4) shows adjusted survival: at 5 years post-CABG, survival was $80 \%$ and $71 \%$ for patients who received an ICD com-

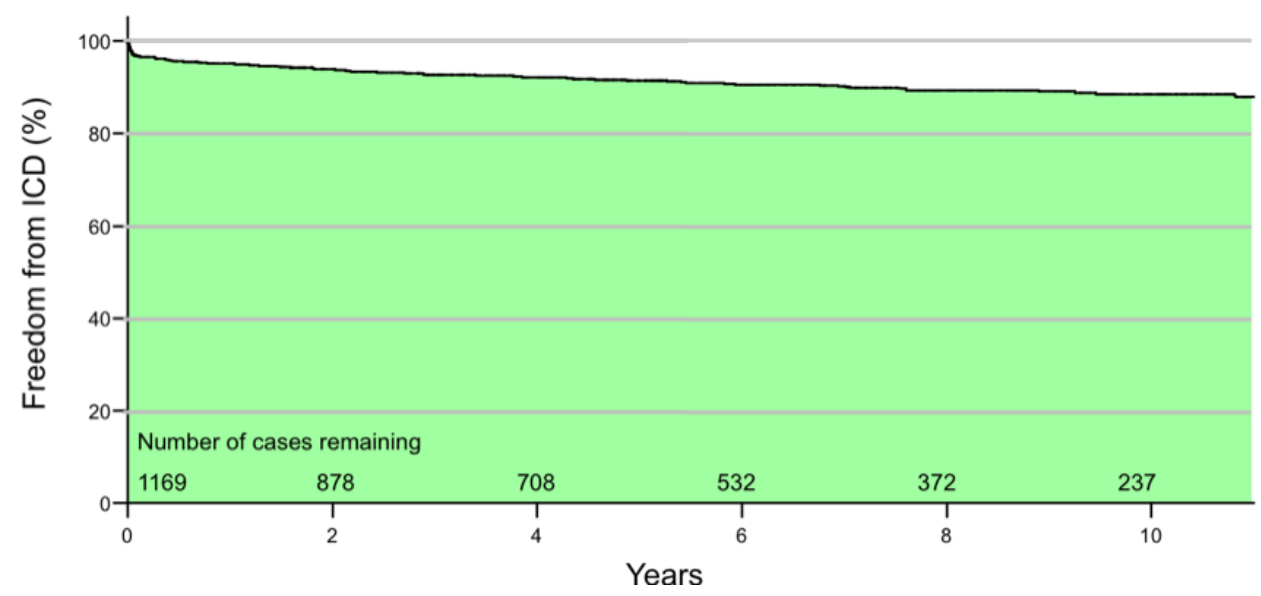

Fig. (1). Freedom from implantable cardioverter defibrillator (ICD) implantation among coronary artery bypass grafting (CABG) patients with left ventricular ejection fraction $(\mathrm{LVEF}) \leq 35 \%$ between March 1, 1995 and June 30, 2008. Incomplete mortality data. After 10 years 237 of 1,169 cases were alive and without an ICD. Freedom from ICD implantation in eligible patients was $95 \%$ at year $1,93 \%$ at year 3, and $91 \%$ at year 5 . The area under the curve illustrates a majority of patients not receiving an ICD during follow-up.

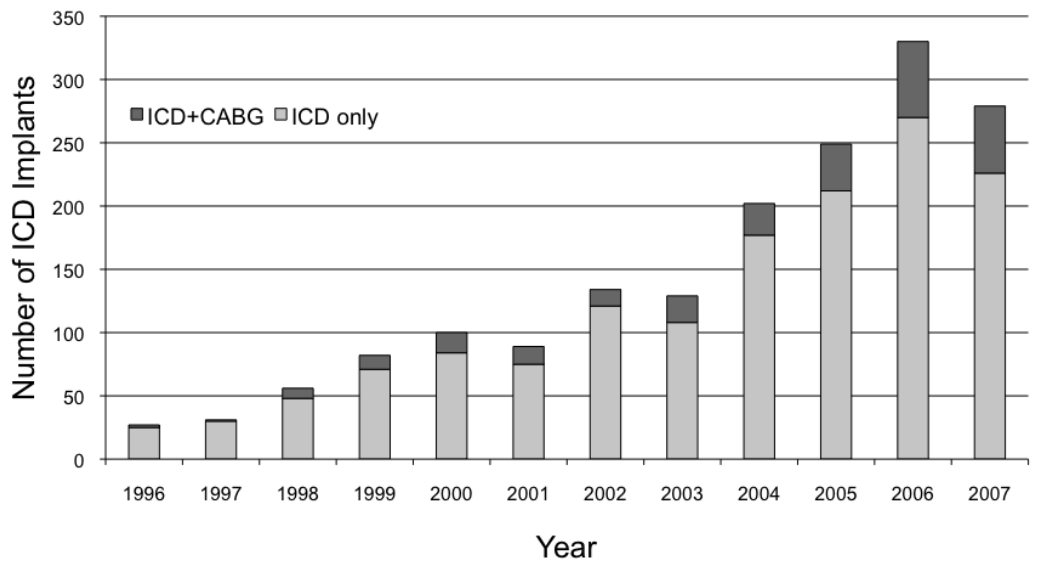

Fig. (2). Annual implantable cardioverter-defibrillator (ICD) implantation rate stratified by ICD only and ICD in patients with a history of coronary artery bypass grafting $(\mathrm{ICD}+\mathrm{CABG})$ procedures. 
Table 2. Risk Adjusted Odds Ratio Estimate of Variable Impact on Future ICD Implantation

\begin{tabular}{|l|l|l|l|}
\hline Patient Characteristic & OR Estimate & 95\% CI & P-Value \\
\hline \hline Arrhythmia & 7.4 & $4.4-12.2$ & $<0.01$ \\
\hline Urgent or Emergent Status & 0.5 & $0.2-0.9$ & 0.01 \\
\hline Age $>70$ & 0.5 & $0.3-0.8$ & $<0.01$ \\
\hline Female Gender & 0.2 & $0.1-0.6$ & $<0.01$ \\
\hline COPD & 0.5 & $0.3-0.8$ & $<0.01$ \\
\hline
\end{tabular}

COPD - chronic obstructive pulmonary disease. ROC $78 \%$.

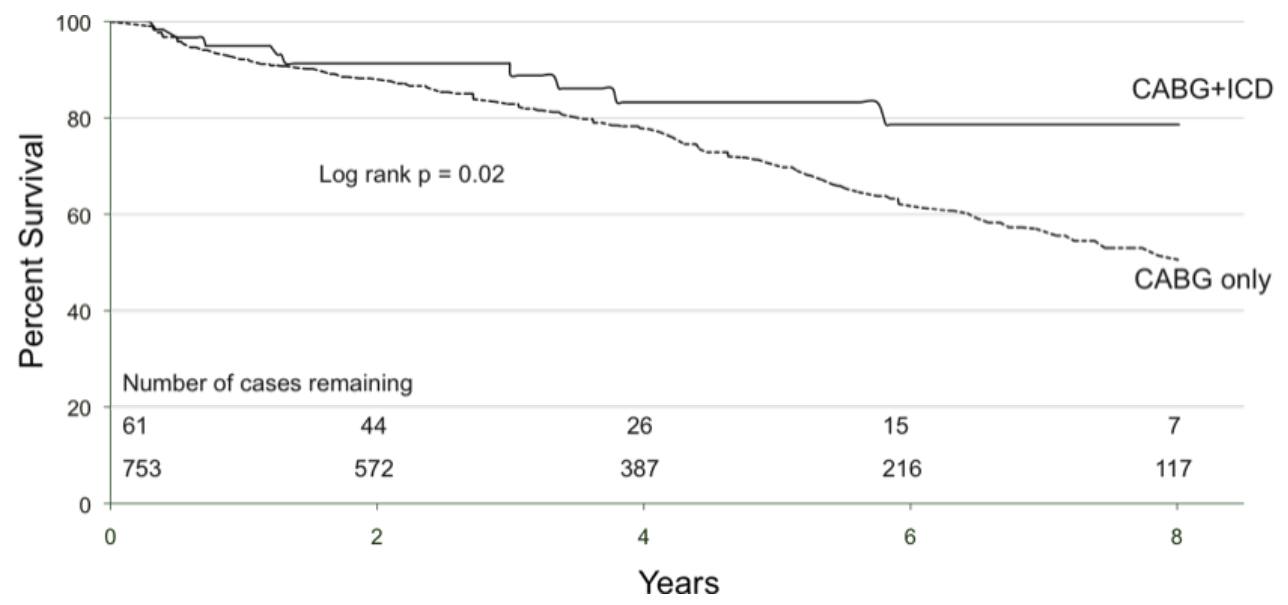

Fig. (3). Kaplan Meier survival curves for implantable cardioverter-defibrillator (ICD) eligible coronary artery bypass graft (CABG) patients who received an ICD post-op ('CABG+ICD') and for those who do not ('CABG only' curve). At 5 years $83 \%$ of CABG+ICD patients are alive compared to $70 \%$ of $\mathrm{CABG}$ only. This represents a relative risk reduction of $45 \%$.

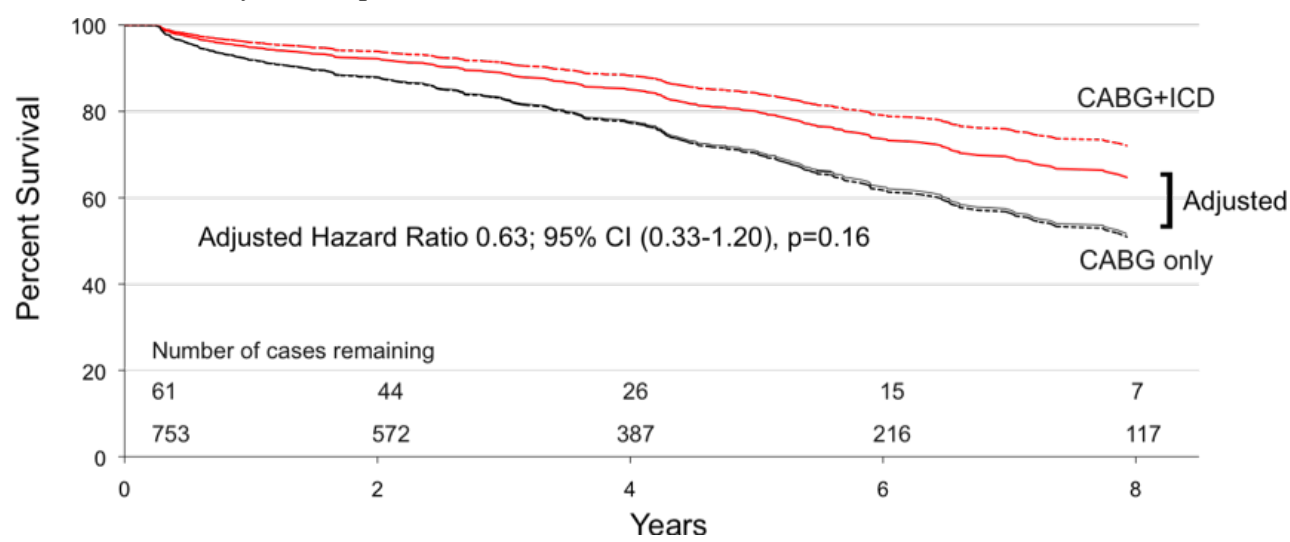

Fig. (4). Adjusted Cox proportional hazard model of survival in eligible patients who received ICD therapy ('CABG+ICD' curve) compared to those that did not ('CABG only' curve). Adjusted Hazard Ratio 0.63; 95\% CI (0.33-1.20).

pared to those who do not, respectively, according to the mean of covariates method which failed to reach significance.

As a data validation step, a chart review of 80 consecutive ICD eligible CABG patients between 2006-2007 revealed that only 18 patients $(22.5 \%)$ underwent EF assessment $\approx 3$ months after surgical revascularization despite current recommendations. Of these 18 patients, 10 had persistent LV dysfunction with $\mathrm{EF} \leq 35 \%$ suggesting that a significant proportion of revascularized patients may have insufficient improvement in $\mathrm{EF}$ and therefore remain eligible (one of our criteria for eligibility). Based on these findings one could predict that more than 900 patients may not have had appropriate follow-up EF evaluation with as many as half of these patients exhibiting persistent LVEF dysfunction and as such remaining ICD eligible.

\section{DISCUSSION}

In the present study we evaluated the utilization of ICD therapy in patients with ischemic cardiomyopathy, defined as those patients with a history of ischemic heart disease and an LVEF $\leq 35 \%$, based on currently published guidelines [14$15,21]$. Our entry criteria for ischemic heart disease, was based on having undergone surgical revascularization 
(CABG) in Nova Scotia with patients identified using The Maritime Heart Center Cardiac Surgery Registry Database. This was not a review of all ischemic cardiomyopathy patients triaged at our institution but was specific to patients undergoing surgical revascularization. During our study period extending from 1995-2008, a total of 1,169 consecutive patients were identified as ICD eligible. In this large group of eligible patients with $100 \%$ follow-up, only 101 (8.6\%) patients received an ICD post surgical revascularization suggesting low rates of ICD utilization. Freedom from ICD in this patient population was $95 \%$ at year $1,93 \%$ at year 3, and $91 \%$ at year 5 of follow-up illustrating the low rates of utilization over time. Similar finding have been suggested by others who claim that ICD therapy is still vastly underutilized among eligible candidates for primary prevention of lethal arrhythmias [16-19].

The retrospective nature of the study resulted in the unavoidable limitation that LVEF used to define eligibility was determined prior to revascularization. This limitation is based on evidence from published series that LVEF postCABG should improve, however, these findings may not necessarily be present or sufficient in all patients [22-24]. We have tried to address this limitation by retrospectively looking at a group of consecutive ICD eligible patients from 2006 - 2007 and evaluated the documented changes in LVEF post-CABG. Using this approach we found that as few as $23 \%$ of patients underwent an EF assessment postCABG with less than half demonstrating improvement in LVEF above $35 \%$. We speculate that if these findings were to be extrapolated to the remainder of the patient population, it is likely that a large proportion of patients included in the present study would remain ICD eligible post revascularization. Furthermore, our data suggests that inadequate followup evaluation of EF post revascularization may be one of the major reasons why patients are not considered for ICD therapy. The rationale for looking at patient records from a small group of consecutive patients $(n=80)$ was based on data access limitations. The present study made use of large administrative data sets, which forbid access to individual patient information a pre-requisite for data encryption and linkage between data sets (confidentiality agreement for MHCPHRU linkage). Finally, while somewhat liberal, our approach in defining patient eligibility using administrative data in a large group of patients has been used by others supporting our observations and approach [14].

The results of the CABG-Patch trial would suggest that underutilization of prophylactic ICD implantation among CABG patients with impaired LVEF is justified [25]. Briefly, this randomized control trial found no evidence of survival benefit when ICD implantation occurred at the time of CABG surgery in a population of eligible patients similar to those we examined. However, patients in the CABG-Patch trial were selected for ICD implantation on the basis of ' $a b$ normal signal averaged ECG changes' compared to inducible, sustained ventricular tachyarrhyhthmias in subsequent studies. After further investigation it was also found that a 45\% reduction in arrhythmic deaths after ICD implantation in the CABG-Patch trial was obscured by a high rate of nonarrhythmic mortality [26]. Since the mid-1990s several other primary prevention trials have shown significant mortality risk reduction with ICD therapy in this patient population including the Multicenter Automatic Defibrillator Implantation Trial (MADIT) [5-9]. While our risk adjusted survival analysis was unable to support a survival advantage to ICD therapy once should note that we were also underpowered to make the conclusion that ICD are not beneficial. Therefore, it remains possible that ICD therapy in our patient population could provide long-term survival benefit.

Using fully adjusted logistic regression analysis we were able to identify a single independent predictor of ICD utilization, which was a history of arrhythmic events prior to revascularization. This finding is in keeping with secondary prevention guidelines [11-15, 21]. We also identified 4 independent variables that negatively predicted future ICD implantation in our population of eligible patients. Our data suggests that female gender, patients over the age of 70, patients requiring emergent surgery at the time of CABG, and a history of severe lung disease were independently associated with a significantly reduced likelihood of ever receiving an ICD.

We are not the first to observe low ICD utilization among eligible women [16-19]. While some have speculated that women with ischemic disease have a lower incidence of SCD than men, explaining the lower rates of utilization, it has been shown that men and women with impaired left ventricular function and ischemic disease receive similar survival benefit from ICD implantation [17, 19, 27]. Others have suggested that women are generally older then males at time of presentation, yet our multivariate analysis provided evidence that age alone cannot explain the discrepancy.

Several factors that are difficult to adjust might have contributed to the low utilization of ICD therapy in elderly patients. Current guidelines suggest that ICD devices should be implanted in those patients who meet the criteria of impaired LVEF post-CABG and are expected to survive with adequate functional status for more than a year [12-15]. However, patients over 70 who present with ventricular dysfunction have the potential to be perceived by physicians as less capable of attaining adequate functional status in the future. As a result, device cost and resource allocation may be a plausible factor in the underutilization of ICD therapy in this group.

The present study was not designed to identify specific barriers to access. The evolution of clinical recommendations and practice guidelines between 1995 and 2008 make this nearly impossible in our study population. Unfortunately, given the small number of patients receiving an ICD, it was also difficult to obtain meaningful data from examining utilization stratified by ICD recommendation eras. Nevertheless, we provide strong evidence that ICD utilization is low post-CABG surgery in patients with ischemic cardiomyopathy. Furthermore, our findings suggest that a lack of follow-up assessment of LVEF post surgical revascularization (fewer than 23\% assessed) may explain why patients are not considered eligible for ICD despite clear guidelines suggesting follow-up evaluation.

\section{CONCLUSIONS}

Despite the above limitations we provide data from a large Canadian registry looking at patients with coronary artery disease and impaired LV function, many of which are 
eligible for ICD implantation post surgical revascularization. We provide valuable every-day clinical practice information suggesting low rates of ICD utilization $(<10 \%)$ with age and gender inequalities among several sub-groups of ICD eligible patients. Based on our findings, LVEF assessment post revascularization may be as low at $23 \%$ and may represent a mechanism explaining why patients are not considered for ICD therapy.

\section{ABBREVIATIONS}

$\begin{array}{ll}\text { SCD } & =\text { Sudden Cardiac Death } \\ \text { ICD } & =\text { Implantable cardioverter-defibrillator } \\ \text { VF } & =\text { Ventricular Fibrillation } \\ \text { VT } & =\text { Canadian Cardiovascular Society } \\ \text { CCS } & =\text { Left Ventricular Ejection Fraction } \\ \text { LVEF } & =\text { Myocardial Infarction } \\ \text { MI } & =\text { New York Heart Association } \\ \text { NYHA } & =\text { Coronary Artery Bypass Grafting } \\ \text { CABG } & =\text { Queen Elizabeth II Health Sciences Center } \\ \text { QEII HSC } & =\text { Electrophysiology Specialist } \\ \text { EPS } & =\text { Population Health Research Unit } \\ \text { PHRU } & =\text { Canadian Institute for Health Information } \\ \text { CIHI } & =\text { Discharge Abstract Database } \\ \text { DAD } & =\text { Multicenter Automatic Defibrillator } \\ \text { MADIT } & \end{array}$

\section{COMPETING INTERESTS}

The authors of this manuscript have no competing interests to disclose

\section{AUTHORS CONTRIBUTIONS}

RK carried out the study design and drafted the manuscript. $\mathrm{KB}$ carried out the statistical analysis. $\mathrm{OH}$ participated in the data collection and participated in drafting the manuscript. MB was involved with the study design and editing manuscript. JFL conceived of the study, and participated in its design and coordination and helped to draft the manuscript. All authors read and approved the final manuscript.

\section{REFERENCES}

[1] DiMarco JP. Implantable cardioverter-defibrillators. N Engl J Med 2003; 349: 1836-47.

[2] Goldenberg I, Moss AJ. Implantable device therapy. Prog Cardiovasc Dis 2008; 50: 449-4.

[3] Turakhia M, Tseng ZH. Sudden cardiac death: epidemiology, mechanisms, and therapy. Curr Probl Cardiol 2007; 32: 501-46.

[4] Connolly SJ, Hallstrom AP, Cappato R, et al. Meta-analysis of the implantable cardioverter defibrillator secondary prevention trials. AVID, CASH and CIDS studies. Antiarrhythmics vs Implantable Defibrillator study. Cardiac Arrest Study Hamburg . Canadian Implantable Defibrillator Study. Eur Heart J 2000; 21: 2071-8.

[5] Moss AJ, Hall WJ, Cannom DS, et al. Improved survival with an implanted defibrillator in patients with coronary disease at high risk for ventricular arrhythmia. Multicenter Automatic Defibrillator
Implantation Trial Investigators. N Engl J Med 1996; 335: 193340.

[6] Moss AJ, Zareba W, Hall WJ, et al. Prophylactic implantation of a defibrillator in patients with myocardial infarction and reduced ejection fraction. N Engl J Med 2002; 346: 877-83.

[7] Goldenberg I, Moss AJ, McNitt S, et al. Time dependence of defibrillator benefit after coronary revascularization in the Multicenter Automatic Defibrillator Implantation Trial (MADIT)-II. J Am Coll Cardiol 2006; 47: 1811-7.

[8] Al-Khatib SM, Hellkamp AS, Lee KL, et al. Implantable cardioverter defibrillator therapy in patients with prior coronary revascularization in the Sudden Cardiac Death in Heart Failure Trial (SCD-HeFT). J Cardiovasc Electrophysiol 2008; 19: 1059-65.

[9] Hohnloser SH, Kuck KH, Dorian P, et al. Prophylactic use of an implantable cardioverter-defibrillator after acute myocardial infarction. N Engl J Med 2004; 351: 2481-8.

[10] Kadish A, Dyer A, Daubert JP, et al. Prophylactic defibrillator implantation in patients with nonischemic dilated cardiomyopathy. N Engl J Med 2004; 350: 2151-8.

[11] Arnold JM, Liu P, Demers C, et al. Canadian Cardiovascular Society consensus conference recommendations on heart failure 2006: diagnosis and management. Can J Cardiol 2006; 22: 23-45.

[12] Zipes DP, Camm AJ, Borggrefe M, et al. ACC/AHA/ESC 2006 guidelines for management of patients with ventricular arrhythmias and the prevention of sudden cardiac death: a report of the American College of Cardiology/American Heart Association Task Force and the European Society of Cardiology Committee for Practice Guidelines (Writing Committee to Develop Guidelines for Management of Patients With Ventricular Arrhythmias and the Prevention of Sudden Cardiac Death). J Am Coll Cardiol 2006; 48: e247346.

[13] Epstein AE, DiMarco JP, Ellenbogen KA, et al. ACC/AHA/HRS 2008 Guidelines for Device-Based Therapy of Cardiac Rhythm Abnormalities: a report of the American College of Cardiology/American Heart Association Task Force on Practice Guidelines (Writing Committee to Revise the ACC/AHA/NASPE 2002 Guideline Update for Implantation of Cardiac Pacemakers and Antiarrhythmia Devices) developed in collaboration with the American Association for Thoracic Surgery and Society of Thoracic Surgeons. J Am Coll Cardiol 2008; 51: e1-62.

[14] Howlett JG, McKelvie RS, Arnold JM, et al. Canadian Cardiovascular Society Consensus Conference guidelines on heart failure, update 2009: diagnosis and management of right-sided heart failure, myocarditis, device therapy and recent important clinical trials. Can J Cardiol 2009; 25: 85-105.

[15] Gregoratos G, Abrams J, Epstein AE, et al. ACC/AHA/NASPE 2002 Guideline Update for Implantation of Cardiac Pacemakers and Antiarrhythmia Devices--summary article: a report of the American College of Cardiology/American Heart Association Task Force on Practice Guidelines (ACC/AHA/NASPE Committee to Update the 1998 Pacemaker Guidelines). J Am Coll Cardiol 2002; 40: 1703-19.

[16] Udell JA, Juurlink DN, Kopp A, Lee DS, Tu JV, Mamdani MM. Inequitable distribution of implantable cardioverter defibrillators in Ontario. Int J Technol Assess Health Care 2007; 23: 354-61.

[17] Curtis LH, Al-Khatib SM, Shea AM, Hammill BG, Hernandez AF, Schulman KA. Sex differences in the use of implantable cardioverter-defibrillators for primary and secondary prevention of sudden cardiac death. JAMA 2007; 298: 1517-24.

[18] Hernandez AF, Fonarow GC, Liang L, et al. Sex and racial differences in the use of implantable cardioverter-defibrillators among patients hospitalized with heart failure. JAMA 2007; 298: 1525-32.

[19] Yarnoz MJ, Curtis AB. Sex-based differences in cardiac resynchronization therapy and implantable cardioverter defibrillator therapies: effectiveness and use. Cardiol Rev 2006; 14: 292-8.

[20] Legare JF, MacLean A, Buth KJ, Sullivan JA. Assessing the risk of waiting for coronary artery bypass graft surgery among patients with stenosis of the left main coronary artery. CMAJ 2005; 173: $371-5$.

[21] Tang AS, Ross H, Simpson CS, et al. Canadian Cardiovascular Society/Canadian Heart Rhythm Society position paper on implantable cardioverter defibrillator use in Canada. Can J Cardiol 2005; 21 Suppl A: 11A-8A.

[22] Soliman Hamad MA, Tan ME, van Straten AH, van Zundert AA, Schonberger JP. Long-term results of coronary artery bypass graft- 
ing in patients with left ventricular dysfunction. Ann Thorac Surg 2008; 85: 488-93.

[23] Bax JJ, Poldermans D, Elhendy A, et al. Improvement of left ventricular ejection fraction, heart failure symptoms and prognosis after revascularization in patients with chronic coronary artery disease and viable myocardium detected by dobutamine stress echocardiography. J Am Coll Cardiol 1999; 34: 163-9.

[24] Elefteriades JA, Tolis G Jr, Levi E, Mills LK, Zaret BL. Coronary artery bypass grafting in severe left ventricular dysfunction: excellent survival with improved ejection fraction and functional state. $\mathbf{J}$ Am Coll Cardiol 1993; 22: 1411-7.

[25] Bigger JT Jr. Prophylactic use of implanted cardiac defibrillators in patients at high risk for ventricular arrhythmias after coronary- artery bypass graft surgery. Coronary Artery Bypass Graft (CABG) Patch Trial Investigators. N Engl J Med 1997; 337: 1569-75.

[26] Bigger JT Jr, Whang W, Rottman JN, et al. Mechanisms of death in the CABG Patch trial: a randomized trial of implantable cardiac defibrillator prophylaxis in patients at high risk of death after coronary artery bypass graft surgery. Circulation 1999; 99: 1416-21.

[27] Zareba W, Moss AJ, Jackson Hall W, et al. Clinical course and implantable cardioverter defibrillator therapy in postinfarction women with severe left ventricular dysfunction. J Cardiovasc Electrophysiol 2005; 16: 1265-70.

(C) Kelly et al.; Licensee Bentham Open.

This is an open access article licensed under the terms of the Creative Commons Attribution Non-Commercial License (http://creativecommons.org/licenses/ by-nc/3.0/) which permits unrestricted, non-commercial use, distribution and reproduction in any medium, provided the work is properly cited. 